Revue de droit comparé du travail et de la sécurité sociale

3 | 2017

Le travail dans l'économie informelle, un défi pour le droit social

\title{
Réflexion sur les travailleurs de l'économie informelle dans la communauté de développement de l'Afrique australe (SADC)
}

Elmarie Fourie

\section{OpenEdition}

Journals

Édition électronique

URL : https://journals.openedition.org/rdctss/340

DOI : $10.4000 /$ rdctss.340

ISSN : 2262-9815

Éditeur

Centre de droit comparé du travail et de la sécurité sociale

Édition imprimée

Date de publication : 1 septembre 2017

Pagination : 44-64

ISSN : $2117-4350$

Référence électronique

Elmarie Fourie, "Réflexion sur les travailleurs de l'économie informelle dans la communauté de développement de l'Afrique australe (SADC) ", Revue de droit comparé du travail et de la sécurité sociale [En ligne], 3 | 2017, mis en ligne le 01 novembre 2018, consulté le 04 décembre 2021. URL : http:// journals.openedition.org/rdctss/340 ; DOI : https://doi.org/10.4000/rdctss.340

\section{(c) $($ ) $(9)$}

Revue de droit comparé du travail et de la sécurité sociale est mise à disposition selon les termes de la Licence Creative Commons Attribution - Pas d'Utilisation Commerciale - Pas de Modification 4.0 International. 


\section{RÉFLEXION SUR LES TRAVAILLEURS}

DE L'ÉCONOMIE INFORMELLE DANS LA

COMMUNAUTÉ DE DÉVELOPPEMENT DE

L'AfrIQUE AUSTRALE (SADC)

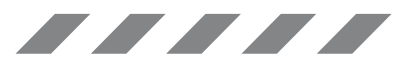

\section{ABSTRACT}

This contribution recognizes that the informal economy is growing phenomenon in developing countries and an increasing reality. Labour and social protection measures were designed to protect those in the traditional employment relationship and is inadequate to protect vulnerable workers in the informal economy. If labour law cannot meet this challenge its function and impact will become negligible in the new world of work. International instruments do exist, however low ratification rates remain problematic. Trade union representation of these workers remain low and is of the utmost importance that these workers mobilize to improve their working conditions. It has become necessary to explore various workers organizations, other than trade unions, to facilitate the needs of these workers.

KEYWORDS: Informal economy, vulnerable, labour and social protection, international and regional regulation, voice and representation.

\section{RÉSUMÉ}

Le présent article part du constat que l'économie informelle occupe une place importante dans les pays en développement, et que ce phénomène s'intensifie. Les mesures existantes en matière de protection sociale et de droit du travail ont été conçues pour protéger les personnes engagées dans une relation de travail traditionnelle et ne sont pas adaptées aux travailleurs vulnérables de l'économie informelle. Si le droit du travail ne parvient pas à surmonter cette difficulté, il deviendra inopérant. Si des instruments juridiques ont bien été adoptés à l'échelle internationale, leur taux de ratification demeure toutefois insuffisant. Les travailleurs de l'économie informelle ne jouissent que d'une faible représentation syndicale, et ils doivent impérativement se mobiliser pour améliorer leurs conditions de travail. Il est essentiel, aujourd'hui, d'envisager le recours à différentes formes d'organisation des travailleurs, outre les syndicats, pour satisfaire à leurs besoins.

MOTS CLÉS: ÉConomie informelle, vulnérabilité, protection sociale et droit du travail, réglementation internationale et régionale, expression et représentation. 


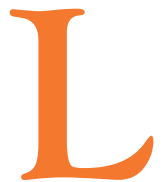

es spécialistes du droit du travail du monde entier débattent actuellement de la fonction et de l'avenir de leur discipline, ainsi que de la nécessité d'en reconsidérer la raison d'être. Le droit du travail fait l'objet de vives critiques en raison de son incapacité à protéger les travailleurs les plus vulnérables, y compris ceux de l'économie informelle, et parce qu'il «favorise les salariés occupant une bonne place, au détriment des moins privilégiés ${ }^{01}$. Le travail informel est aujourd'hui une réalité, reconnue par un nombre toujours croissant d'acteurs $^{02}$. En Afrique du Sud, tout comme dans d'autres pays en développement, les travailleurs de l'économie informelle ne bénéficient pas de protections suffisantes sur le plan social, le droit du travail de ces pays étant fondé sur la relation employeur/salarié traditionnelle. L'existence de l'économie informelle est indéniable; elle représente même $66 \%$ des emplois non agricoles en Afrique subsaharienne ${ }^{03}$.

Ces travailleurs ne sont ni reconnus, ni protégés, ni même régis par le droit du travail ou les mesures de protection sociale, et peuvent présenter différents degrés de «dépendance» et de «vulnérabilité ${ }^{04}$. Le Rapport mondial de l'Organisation internationale du Travail (OIT) sur la protection sociale $2014-2015^{05}$ estime que $73 \%$ de la population mondiale vit encore sans couverture sociale adaptée, avec des droits fondamentaux qui ne sont souvent que partiellement - voire pas du tout - respectés.

Dans cette contribution, la définition retenue de l'économie informelle englobe toutes les activités économiques des travailleurs et des unités économiques qui - en droit ou en pratique - ne sont pas couvertes ou sont insuffisamment couvertes par des dispositions formelles ${ }^{06}$. Cette définition et cette discussion excluent toutes les activités illicites ${ }^{07}$.

01 G. Davidov et B. Langille, « Understanding Labour Law: A Timeless Idea, a Timed-Out Idea, or an Idea whose Time has now come? », in G. Davidov et B. Langille (dir.), The Idea of Labour Law, Oxford University Press, 2011, p. 1

02 Plusieurs facteurs ont contribué à la reconnaissance croissante de la réalité du travail informel : la disponibilité de statistiques améliorées, le Programme de l'OIT pour un travail décent, la création de réseaux internationaux et nationaux au service des travailleurs de l'économie informelle, la prise de conscience du fait que la majorité de ces personnes n'y travaillent pas par choix et l'accent mis sur le rôle joué par la protection sociale en matière de réduction de la pauvreté et de la vulnérabilité de ces populations. Voir également la Recommandation de l'OIT sur les socles de protection sociale de 2012 ; F. Lund, « Extending Social Protection/Social Security to Informal Workers: Pathways to Inclusive Practices », communication présentée lors du Congrès mondial de l'ILERA, Le Cap, 2015.

03 M.A. Chen, 2017, « The Informal Economy in African Cities: Key to Inclusive and Sustainable Urban Development », WIEGO, http//www.wiego.org (consulté le 10 août 2017).

04 Résolution de l'OIT concernant le travail décent et l'économie informelle, 2002, conclusion n 9

05 Rapport de l'OIT sur la protection sociale dans le monde, 2014-2015, Vers la reprise économique, le développement inclusif et la justice sociale, 2014 - 2015.

06 Recommandation n²04 concernant la transition de l'économie informelle vers l'économie formelle, 2015

07 La Recommandation n 204 de 2015 définit les activités illicites en ces termes : « la fourniture de services ou la production, la vente, la possession ou la consommation de biens interdites par la loi, y compris la production et le trafic illicites de stupéfiants, la fabrication et le trafic illicites d'armes à feu, la traite des personnes et le blanchiment d'argent, tels que les définissent les conventions internationales pertinentes. » 
Lorsque l'on étudie la protection des travailleurs informels, il est important d'examiner la conception et la mise en œuvre de solutions innovantes et adaptées, en tenant compte par exemple de la nature des fonctions exercées et des caractéristiques des lieux de travail. Il convient de souligner l'importance de l'expression et de la représentation collectives dans la protection des travailleurs informels, de même que l'existence, à l'échelle nationale comme internationale, d'organisations de travailleurs engagées auprès de ces personnes. Ces organisations prennent la forme d'associations professionnelles, de coopératives, d'organisations non gouvernementales (ONG) ou encore d'organisations à base communautaire. La création de liens entre ces réseaux, que ce soit à l'échelle nationale ou internationale, peut renforcer les pressions politiques exercées pour promouvoir les intérêts de ces travailleurs. Ces organisations font entendre leur voix en adoptant des approches innovantes et des structures diversifiées qui permettent d'améliorer les conditions de travail de leurs membres. Leur capacité de négociation collective en vue d'améliorer ces conditions de travail est extrêmement importante, notamment dans les pays où l'économie informelle côtoie l'économie formelle ${ }^{08}$.

\section{I - Le monde du travail dans les pays en développement}

Dans les pays en développement, l'économie informelle représente la norme plutôt que l'exception. Selon les estimations, plus de $80 \%$ des travailleurs pauvres des pays en développement interviennent dans l'économie informelle et survivent avec moins de 2 dollars par jour ${ }^{09}$. La part du travail informel varie selon les pays de la Communauté de développement d'Afrique australe (SADC); il représente ainsi $33 \%$ des emplois non agricoles en Afrique du Sud, $44 \%$ en Namibie, ou encore $76 \%$ en Tanzanie ${ }^{10}$. Des études révèlent, par ailleurs, que les revenus tirés du travail informel produisent des effets visibles sur la réduction de la pauvreté des ménages démunis ${ }^{11}$. L'économie informelle de l'Afrique du Sud est l'une des moins développées de la région. Dans ce pays, $14 \%$ des revenus proviennent des bénéfices tirés du secteur informel, permettant aux ménages de dépasser le seuil de pauvreté ${ }^{12}$. Le fait que ce revenu contribue à la lutte contre la pauvreté des ménages est particulièrement important pour les décideurs politiques, puisqu'il leur permet de comprendre la valeur du travail dans l'économie informelle. Le régime de protection sociale et du travail de ces travailleurs constitue donc une question primordiale compte tenu du taux de chômage élevé en Afrique du Sud et des effets du secteur informel sur la réduction de la pauvreté des ménages.

08 N. Smit et E. Fourie, « Extending Protection to Atypical Workers, Including Workers in the Informal Economy, in Developing Countries », International Journal of Comparative Labour Law and Industrial Relations, 26, 2010, $\mathrm{n}^{\circ} 1$, p. 43-60.

09 M. Rogan et P. Cichello, « Can Informal Employment Actually Reduce Poverty? », WIEG0, 2017.

10 Les personnes employées dans l'économie informelle sont les suivantes : les travailleurs indépendants, les dirigeants d'entreprises informelles, leurs salariés, les membres de leur famille contribuant à l'entreprise, les membres de coopératives et les salariés occupant des postes informels sans protection sociale ou du travail adaptée. V. J. Vanek et al., « Statistics on the Informal Economy: Definitions, Regional Estimates \& Challenges », document de travail n² 2 WIEGO, 2014.

11 M. Rogan et P. Cichello, « Can Informal Employment Actually Reduce Poverty? », WIEGO Empowering Informal Workers, Securing Livelihoods, 2017.

12 Ibidem. 


\section{L'économie informelLe en Afrique Australe}

Le tableau 1 illustre la situation de la population active en Afrique du Sud en 2017 ( $2^{\mathrm{e}}$ trimestre).

Nombre

\begin{tabular}{|l|l|c|c|c|c|c|}
\hline & Hommes & Femmes & Total & Hommes (\%) & Femmes (\%) & Total \\
\hline $\begin{array}{l}\text { Secteur formel } \\
\text { (non agricole) }\end{array}$ & 6494273 & 4803462 & 11297734 & 57,4 & 42,6 & 100 \\
\hline $\begin{array}{l}\text { Secteur informel } \\
\text { (non agricole) }\end{array}$ & 1759667 & 1049432 & 2809099 & 62,8 & 37,2 & 100 \\
\hline Secteur agricole & 580041 & 264637 & 844678 & 68,4 & 31,6 & 100 \\
\hline Ménages privés & 298065 & 1027935 & 1325999 & 22,4 & 77,6 & 100 \\
\hline
\end{tabular}

Source : Statistics South Africa, enquête sur la population active 2017 ( $2^{\mathrm{e}}$ trimestre $^{13}$.

En 2004, plusieurs postulats concernant les liens entre le caractère informel de l'emploi, le genre et la pauvreté ont été avancés : les pauvres sont plus susceptibles de travailler dans l'économie informelle; l'économie informelle compte plus de femmes pauvres que de femmes non pauvres; les revenus des femmes travaillant dans le secteur informel sont moins élevés que ceux de leurs homologues masculins, et elles sont moins susceptibles d'être organisées et de bénéficier d'une protection sociale ${ }^{14}$. Les femmes continuent d'occuper plus d'emplois précaires que les hommes. Dans les régions en développement, $60 \%$ des femmes avaient un emploi précaire en 2013, contre $54 \%$ des hommes ${ }^{15}$. En Afrique du Sud, tout comme dans d'autres États membres de la SADC, ces femmes vulnérables ne sont protégées par aucune mesure de sécurité sociale adaptée, en raison d'exclusions réglementaires et du parti pris par de nombreux systèmes de sécurité sociale de ces pays en faveur de l'économie formelle ${ }^{16}$.

En 2015, le Forum économique mondial a reconnu que les travailleurs pauvres avaient besoin de filets de sécurité sociale pour pallier les effets des chocs externes et transitoires sur leurs moyens de subsistance, ainsi que d'une assurance sociale visant à réduire les risques associés à un problème de santé, au handicap, à un accident du travail et à la vieillesse. Une reconnaissance appropriée et la régulation des secteurs de l'économie informelle favoriseraient l'éradication de la pauvreté au sein de cette population. La loi ne peut, à elle seule, garantir le principe du travail décent, aussi inclusive la législation sur le travail et aussi étendues les mesures de protection sociale soient-elles ${ }^{17}$.

13 SA Stats, http//www.statsa.gov.za (consulté le 10 août 2017).

14 M.A. Chen, J. Vanek et M. Carr, Mainstreaming Informal Employment and Gender in Poverty Reduction, WIEGO, 2004, p. 30.

15 Nations Unies, Objectifs du Millénaire pour le développement, Rapport 2014, 2014, p. 11. Les plus grandes disparités entre les sexes étaient observées en Afrique du Nord, en Afrique subsaharienne et en Asie de l'Ouest. Au Brésil, l'augmentation du salaire minimum des femmes a entraîné une réduction des écarts de rémunération entre hommes et femmes. Voir ONU-Femmes, Le progrès des femmes dans le monde, 20152016, http://www.progress.unwomen.org/en/2015,2015 (consulté le 10 juin 2017).

16 F. Ebert Stiftung, «Engendering Social Protection Conference Summary Report », Conférence internationale de Lusaka, 22-23 octobre 2012, p. 8.

17 OIT, Decent Work Indicators: Concepts and Definitions Report, 2012. 


\section{II - Le cadre juridique international}

L'OIT a reconnu l'essor des relations de travail atypiques et la nécessité d'apporter une protection en matière de sécurité sociale et de travail à ces travailleurs, à travers l'adoption de conventions et de recommandations spécifiques, la fourniture d'une aide aux micro-entreprises, la mise en place de programmes de promotion de l'élargissement de la protection sociale aux travailleurs du secteur informe ${ }^{18}$, l'appui aux mutuelles de soins de santé et la recherche en matière d'accès à la protection sociale pour les travailleurs atypiques ${ }^{19}$. Le regain d'intérêt pour l'économie informelle pourrait être dû au fait que, dans la plupart des régions en développement, plus de la moitié des emplois non agricoles relèvent du travail informel, ce chiffre atteignant même $82 \%$ des emplois non agricoles en Asie du sud. Il peut provenir également de la reconnaissance mondiale des liens existant entre informalité et croissance d'une part, et entre informalité, pauvreté et inégalités d'autre $\operatorname{part}^{20}$.

En Afrique du Sud, les normes internationales du travail jouent un rôle particulièrement important pour le développement du droit du travail national et agissent comme source d'un droit international coutumier. À partir de 1994, la nouvelle Constitution a reconnu la place prépondérante qu'occupent les normes internationales dans un pays démocratique. L'Afrique du Sud est également redevenue membre de l'OIT le 26 mai 1994 et a depuis ratifié les 8 conventions fondamentales ${ }^{21}$.

La plupart des normes fondamentales du travail s'appliquent à l'ensemble des travailleurs ou contiennent des dispositions qu'il est possible d'étendre à diverses catégories de travailleurs. Par ailleurs, l'OIT a adopté, en 1998, la Déclaration relative aux principes et droits fondamentaux au travail, qui prévoit que les États signataires doivent adopter au minimum les conventions fondamentales. À l'échelle internationale, la tendance est à l'extension de la protection aux travailleurs atypiques, mais le nombre de pays ayant ratifié les conventions pertinentes ${ }^{22}$ demeure faible, de même que la part de travailleurs atypiques dans les pays concernés. L'efficacité de ces conventions en matière de protection des travailleurs atypiques reste donc limitée.

La Recommandation n ${ }^{\circ} 202$ de l'OIT sur les socles de protection sociale (2012) tente de fournir à l'ensemble des travailleurs un régime de sécurité sociale de base leur garantissant au minimum la santé et la dignité. Il est préconisé que les États prévoient des niveaux minima de protection prenant la forme de socles de protection sociale. Les garanties de base en matière de sécurité sociale, comme la couverture médicale, la sécurité de revenu en cas de maladie et les prestations chômage et maternité, peuvent assurer un certain niveau de protection aux travailleurs de l'économie informelle. La Recommandation souligne, par ailleurs, l'importance de la coopération entre les parties prenantes pour assurer l'existence de niveaux minima de protection.

18 Stratégies et techniques contre l'exclusion sociale et la pauvreté (STEP).

19 Voir également la Recommandation n 204 de l'OIT concernant la transition de l'économie informelle vers l'économie formelle, de 2015, et la Recommandation sur les socles de protection sociale, de 2012.

20 M. Chen, «The Informal Economy: Definitions, Theories and Policies», document de travail $n^{\circ} 1$ WIEGO, 2012, p. 2.

21 Voir également D. Du Toit et al., Labour Relations Law : A Comprehensive Guide, Lexis Nexis South

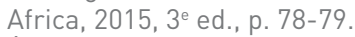

22 À savoir notamment la Convention $n^{\circ} 177$ sur le travail à domicile de 1996 (ratifiée par seulement dix pays); la Convention $n^{0} 189$ sur les travailleuses et travailleurs domestiques de 2011 (celle-ci a été ratifiée par 24 pays) ; la Convention $n^{\circ} 169$ relative aux peuples indigènes et tribaux de 1989. 
L'OIT a adopté le principe du «travail décent». Dans ce cadre, les États membres doivent poursuivre les quatre objectifs suivants : la création d'emplois, le respect des droits fondamentaux des travailleurs, la protection sociale et la représentation collective des travailleurs. Cette notion pourrait servir à donner de l'élan à l'amélioration de la situation précaire des travailleurs de l'économie informelle. Il est important de relier les initiatives en faveur du travail décent aux autres initiatives en matière de protection sociale et de droit du travail. L'OIT s'est engagée à faire du travail décent une réalité pour tous, comme le montre clairement l'adoption de la Convention $n^{\circ} 189$ concernant le travail décent pour les travailleuses et travailleurs domestiques (2011) et sa Recommandation connexe.

\section{A - L'OIT et l'économie informelle}

L'OIT a utilisé pour la première fois le terme «secteur informel» il y a de cela 43 ans ${ }^{23}$. Ce terme décrivait alors les «activités» des travailleurs pauvres, non réglementées et n'assurant pas de protection en matière de sécurité sociale ou de travail. À ce stade, l'OIT reconnaissait déjà l'importance d'une stratégie exhaustive et intégrée permettant de fournir une protection suffisante à cette population. En 2002, l'OIT a affirmé que l'économie informelle était «d'un ordre de grandeur et d'une complexité beaucoup plus grands [qu'auparavant]», et qu'elle ne pouvait plus être considérée comme un phénomène temporaire ou résiduel ${ }^{24}$. Dans son rapport intitulé «Étendre le champ d'application des lois du travail à l'économie informelle», de 2010, l'Organisation a recensé les causes du caractère informel de l'économie, à savoir : une gouvernance faible et des politiques sociales inadaptées et mal conçues et/ou mal mises en œuvre ${ }^{25}$.

Se rendant compte de l'inadéquation du terme «secteur informel», l'OIT a décidé de le remplacer par le terme «économie informelle», défini de manière détaillée dans la Recommandation $n^{\circ} 204$ concernant la transition de l'économie informelle vers l'économie formelle de $2015^{26}$. Il est difficile de donner une définition complète de ce terme, compte tenu des aspects nombreux et hétérogènes de l'économie informelle et des besoins des différentes catégories de travailleurs. Ces derniers présentent toutefois une caractéristique commune : ils ne bénéficient d'aucune protection en matière de sécurité sociale ou de travail, ou bien celle-ci est insuffisante, et ils sont particulièrement vulnérables.

23 OIT, Travail décent et économie informelle, Conférence internationale du Travail, 2002, 90 session.

24 OIT, Travail décent et économie informelle, Conférence internationale du Travail, 2002, $90^{e}$ session, p. 1. Dans les pays en développement, l'économie informelle est souvent celle qui offre le plus d'opportunités d'emploi.

25 Voir le rapport, p. 6

26 Le terme «économie informelle» désigne toutes les activités économiques des travailleurs et des unités économiques qui - en droit ou en pratique - ne sont pas couvertes ou sont insuffisamment couvertes par des dispositions formelles, et ne désigne pas les activités illicites, en particulier la fourniture de services ou la production, la vente, la possession ou la consommation de biens interdites par la loi, y compris la production et le trafic illicites de stupéfiants, la fabrication et le trafic illicites d'armes à feu, la traite des personnes et le blanchiment d'argent, tels que les définissent les conventions internationales pertinentes. 
La Recommandation $n^{\circ} 204$ concernant la transition de l'économie informelle vers l'économie formelle a été adoptée en juin 2015, et propose d'importantes stratégies pour faciliter le passage de l'économie informelle à l'économie formelle, prenant notamment en considération les éléments suivants :

a) la diversité des caractéristiques de l'économie informelle ${ }^{27}$;

b) la spécificité de la situation et des besoins des travailleurs de l'économie informelle ${ }^{28}$;

c) la nécessité de tenir compte de la diversité des situations afin d'élaborer des approches sur mesure ${ }^{29}$;

d) la promotion et la protection effectives des droits humains ${ }^{30}$;

e) la réalisation du travail décent pour tous ;

f) la promotion de l'égalité entre femmes et hommes et la non-discrimination ;

g) et la nécessité de prêter une attention spécifique à certains groupes particulièrement vulnérables, notamment les femmes, les jeunes, les migrants et les personnes âgées.

La Recommandation peut s'avérer très utile pendant le processus de transition, puisqu'elle prend expressément note de la nature diversifiée de l'économie informelle et de l'importance de solutions innovantes et adaptées pour l'extension de la protection aux travailleurs informels. Elle reconnaît que les États membres doivent faire particulièrement attention aux besoins et aux situations des personnes évoluant dans l'économie informelle lors de la mise en place et du maintien de socles nationaux de protection sociale. Elle est un outil précieux lorsque la transition entre l'économie informelle et l'économie formelle devient réalisable et souhaitée. Cette Recommandation ne signifie pas que l'économie informelle sera absorbée par l'économie formelle, mais elle proposera, dans la mesure du possible, des mesures adaptées visant à favoriser la transition.

Le tableau suivant recense les instruments internationaux relatifs à la protection du travail et applicables aux travailleurs de l'économie informelle, qui s'ajoutent aux 8 conventions fondamentales ${ }^{31}$.

27 Cette stratégie reconnaît l'hétérogénéité de l'économie informelle.

28 La nature diversifiée de l'économie informelle signifie que les politiques et stratégies devront tenir compte des besoins de groupes spécifiques de l'économie informelle. Les besoins des travailleurs domestiques, par exemple, ne sont pas les mêmes que ceux des marchands ambulants de l'économie informelle.

29 Cette stratégie reconnaît que les mesures applicables aux travailleurs de l'économie formelle ne seront généralement pas adaptées à ceux de l'économie informelle. Les régimes de sécurité sociale de nombreux pays ont été conçus pour les personnes occupant un emploi formel. L'absence de rapport employeur/ salarié dans l'économie informelle pourrait signifier que le travailleur doit cotiser deux fois plus pour bénéficier d'un tel régime. Or, compte tenu des revenus faibles et irréguliers de nombreux travailleurs de l'économie informelle, leur capacité de contribution sera faible. Voir également G. Mpedi, «Informal Sector : Introduction», in U. Becker, F. Penning et T. Dijkhoff (dir.), International Standard-Setting and Innovations in Social Security, Kluwer, 2013, p. 289. En Zambie, seulement $10 \%$ de la population travaille dans l'économie formelle. Dans ce pays, la couverture sociale est fondée sur un modèle d'assurance sociale qui exclut une large proportion des travailleurs de l'économie informelle. L'adoption d'approches adaptées permettra de satisfaire davantage les besoins spécifiques de ces différents groupes. E. Kalula, N. Okorafor et P. Bamu, « Towards an Effective Regulatory Framework for Labour Rights and Social Protection in South Africa », in D. Banik, Legal Empowerment Agenda, Poverty, Labour and the Informal Economy in Africa, Ashgate, 2011.

30 L'un des principaux éléments de l'autonomisation juridique concerne la possibilité pour ces travailleurs d'exercer leurs droits.

31 La Convention $n^{\circ} 87$ sur la liberté syndicale et la protection du droit syndical de 1948, la Convention $n^{\circ} 98$ sur le droit d'organisation et de négociation collective de 1949 et la Convention $n^{\circ} 111$ concernant la discrimination (emploi et profession) de 1958 ont été ratifiées par l'ensemble des pays de la SADC. La Convention n 100 sur l'égalité de rémunération de 1951 a été ratifiée par l'Afrique du Sud, l'Angola, le Botswana, le Lesotho, Madagascar, le Malawi, Maurice, le Mozambique, la République démocratique du Congo, les Seychelles, le Swaziland, la Tanzanie, la Zambie et le Zimbabwe. 


\begin{tabular}{|c|c|c|}
\hline $\begin{array}{l}\text { Instruments internationaux et travailleurs } \\
\text { de l'économie informelle }\end{array}$ & $\begin{array}{l}\text { Taux de } \\
\text { ratification des } \\
\text { États membres* }\end{array}$ & $\begin{array}{l}\text { Ratification par les États } \\
\text { membres de la SADC }\end{array}$ \\
\hline $\begin{array}{l}\text { Déclaration de l'OIT relative aux principes et droits fon- } \\
\text { damentaux au travail, } 1998 \\
\text { Elle appelle à l'application universelle des droits fon- } \\
\text { damentaux et reconnaît la situation vulnérable des tra- } \\
\text { vailleurs de l'économie informelle }\end{array}$ & $\begin{array}{l}\text { Non sujette à } \\
\text { ratification }\end{array}$ & s.o. \\
\hline $\begin{array}{l}\text { Recommandation n¹98 sur la relation de travail, } 2006 \\
\text { Elle fournit des conseils sur ce qu'elle appelle le } \\
\text { «travail déguisé », qui touche souvent les travailleurs } \\
\text { de l'économie informelle, comme les travailleurs à } \\
\text { domicile. }\end{array}$ & $\begin{array}{l}\text { Non sujette à } \\
\text { ratification }\end{array}$ & s.o. \\
\hline $\begin{array}{l}\text { Convention n } 177 \text { sur le travail à domicile, } 1996 \\
\text { Il s'agit d'un instrument international consacré à } \\
\text { une certaine catégorie de travailleurs de l'économie } \\
\text { informelle, qui promeut l'égalité de traitement entre } \\
\text { les travailleurs à domicile et les autres travailleurs } \\
\text { salariés. }\end{array}$ & 10 & $\begin{array}{l}\text { Aucun État membre de la SADC ne l'a } \\
\text { ratifiée à l'heure actuelle. }\end{array}$ \\
\hline $\begin{array}{l}\text { Convention n } 189 \text { sur les travailleuses et travailleurs } \\
\text { domestiques, } 2011 \text { (voir également la Recommandation } \\
n^{\circ} 201 \text { de } 2011 \text { ) } \\
\text { Elle vise à garantir un travail décent à plus de } \\
67 \text { millions de travailleurs domestiques dans le monde. } \\
\text { Elle constitue un bon exemple de convention contenant } \\
\text { des normes spécifiques à un groupe de travailleurs de } \\
\text { l'économie informelle. }\end{array}$ & 24 & $\begin{array}{l}\text { L'Afrique du Sud et Maurice ont ratifié la } \\
\text { Convention, mais l'Afrique du Sud ne respecte } \\
\text { pas ses obligations internationales à cet } \\
\text { égard. En effet, les travailleurs domestiques } \\
\text { de ce pays ne jouissent pas d'une protection } \\
\text { adaptée, puisqu'ils sont exclus des } \\
\text { prestations pour accident du travail. À } \\
\text { Maurice, la ratification de la Convention a } \\
\text { permis à } 50000 \text { travailleurs et travailleuses } \\
\text { domestiques d'accéder à un fonds de pension } \\
\text { national et à des allocations chômage**. }\end{array}$ \\
\hline $\begin{array}{l}\text { Convention n } 156 \text { sur les travailleurs ayant des res- } \\
\text { ponsabilités familiales, } 1981 \text { (voir également la Recom- } \\
\text { mandation n } 165 \text { de 1981) } \\
\text { Elle s'applique à l'ensemble des travailleurs, y compris } \\
\text { ceux de l'économie informelle. }\end{array}$ & 44 & Maurice \\
\hline $\begin{array}{l}\text { Convention } n^{\circ} 141 \text { sur les organisations de travail- } \\
\text { leurs ruraux, 1975 (voir également la Recommandation } \\
n^{\circ} 149 \text { de 1975). } \\
\text { Elle encourage les travailleurs ruraux à créer des orga- } \\
\text { nisations visant à protéger et promouvoir leurs intérêts. }\end{array}$ & 40 & Zambie \\
\hline $\begin{array}{l}\text { Convention } n^{\circ} 169 \text { relative aux peuples indigènes et } \\
\text { tribaux, } 1989 \\
\text { Les membres de peuples indigènes et tribaux sont sou- } \\
\text { vent actifs dans l'économie informelle. }\end{array}$ & 22 & \\
\hline $\begin{array}{l}\text { Convention } n^{\circ} 81 \text { sur l'inspection du travail, } 1947 \text { (voir } \\
\text { également la Recommandation } n^{\circ} 81 \text { de 1947) } \\
\text { L'inspection du travail joue un rôle important pour } \\
\text { garantir l'application des lois vis-à-vis des travailleurs } \\
\text { de l'économie informelle, tels que les travailleurs et } \\
\text { travailleuses domestiques. }\end{array}$ & 145 & $\begin{array}{l}\text { Afrique du Sud, Angola, Lesotho, Madagas- } \\
\text { car, Malawi, Maurice, Mozambique, Répu- } \\
\text { blique démocratique du Congo, Seychelles, } \\
\text { Tanzanie, Zambie et Zimbabwe. Bien que de } \\
\text { nombreux pays de la SADC aient ratifié cette } \\
\text { convention, son exécution pose encore pro- } \\
\text { blème en raison d'un manque de formation } \\
\text { et d'un nombre insuffisant d'inspecteurs } \\
\text { dans ces pays. }\end{array}$ \\
\hline
\end{tabular}

*OIT Normlex, http://www.ilo.org/dyn/normlex/fr/f?p=NORMLEXPUB:1:0::NO::: (consulté le 4 septembre 2017).

**WIEGO, http://www.wiego.org (consulté le 4 septembre 2017). 


\section{B - L'OIT, la sécurité sociale et l'économie informelle}

Le rapport de l'OIT sur le travail décent de 1999 mettait en lumière le nombre élevé de travailleurs dénués de protection sociale dans l'économie mondiale et une campagne pour étendre cette protection dans les pays en développement a alors vu le jour. En 2001, à travers le concept de travail décent, la Conférence internationale du travail a privilégié la voie de l'extension de la couverture sociale et a souligné l'importance de l'intégration des politiques sociales et d'emploi dans les stratégies nationales ${ }^{32}$.

En 2011, deux problèmes principaux ont été identifiés concernant les instruments de sécurité sociale en vigueur, à savoir le fait que certaines dispositions étaient obsolètes et que ces instruments ne s'alignaient pas sur l'objectif de l'OIT visant à fournir un accès universel à la sécurité sociale. En effet, de nombreux travailleurs de l'économie informelle sont exclus de la protection compte tenu du fait que les règles applicables en matière de sécurité sociale sont fondées sur le paradigme traditionnel de l'emploi à plein temps ${ }^{33}$.

L'exclusion des personnes évoluant en dehors de l'économie formelle, qui continuaient de vivre dans la pauvreté et sans protection sociale adaptée, a placé l'extension de la couverture à ces travailleurs «au cœur du mandat et de la mission de l'OIT » ${ }^{34}$. D'après la définition de l'OIT, les domaines couverts par la protection sociale incluent la sécurité sociale, les conditions de travail, la sécurité au travail, mais aussi les migrations de travail et les questions relatives au $\mathrm{VIH} / \mathrm{sida}$ sur les lieux de travail ${ }^{35}$. Les socles de protection sociale se définissent comme des ensembles de garanties fondamentales de sécurité sociale qui assurent une protection visant à prévenir ou atténuer la pauvreté, la vulnérabilité et l'exclusion sociale ${ }^{36}$. L'extension de la protection sociale aux travailleurs est essentielle pour concrétiser le droit fondamental à la sécurité sociale. La portée limitée des régimes de protection sociale et l'exclusion expresse des travailleurs de l'économie informelle peuvent souvent être imputées à des facteurs tels que le parti pris des régimes d'assurance sociale en faveur du secteur formel, l'existence d'un rapport employeur/ salarié, la faible capacité de contribution ou encore l'incompatibilité des besoins prioritaires ${ }^{37}$.

32 OIT, Étude d'ensemble 2011 concernant les instruments relatifs à la sécurité sociale à la lumière de la Déclaration de 2008 sur la justice sociale pour une mondialisation équitable, Conférence internationale du travail, $100^{\mathrm{e}}$ session, p. 8.

33 OIT, Étude d'ensemble 2011 concernant les instruments relatifs à la sécurité sociale à la lumière de la Déclaration de 2008 sur la justice sociale pour une mondialisation équitable, Conférence internationale du travail, $100^{e}$ session, p. 21. U. Becker, F. Pennings et T. Dijkhoff (dir.), International Standard Setting and Innovations in Social Security, 2013, Kluwer Law International, Pays-Bas, p. 26.

34 OIT, 2011, Etude d'ensemble 2011 concernant les instruments relatifs à la sécurité sociale à la lumière de la Déclaration de 2008 sur la justice sociale pour une mondialisation équitable, Conférence internationale du Travail, $100^{\mathrm{e}}$ session, p. 14.

35 Protection sociale de l'OIT (Caraïbes). Cf. www.ilo.org/Caribbean/areas of work/protection sociale (consulté le 23 octobre 2017).

36 G. Rodgers, E. Lee, L. Swepsten et J. Van Deale, L'Organisation internationale du Travail et la quête de justice sociale, 1919-2009, 2009, OIT, Genève. Le préambule de la Constitution de l'OIT reconnaît la nécessité d'améliorer les conditions de travail grâce à la mise en place de mesures de prévention du chômage et de protection des travailleurs contre la maladie et les accidents du travail, ainsi qu'à la fourniture de prestations de retraite. La Déclaration de Philadelphie reconnaît la sécurité sociale comme un principe fondamental du travail. OIT, 2011, Etude d'ensemble 2011 concernant les instruments relatifs à la sécurité sociale à la lumière de la Déclaration de 2008 sur la justice sociale pour une mondialisation équitable, Conférence internationale du Travail, $100^{\circledR}$ session, p. 7.

37 G. Mpedi, «Informal Sector : Introduction », in U. Becker, F. Penning et T. Dijkhoff (dir.), International Standard-Setting and Innovations in Social Security, 2013, Kluwer Law International, p. 289-290. 
La stratégie de l'OIT en matière de couverture sociale universelle comprend une dimension horizontale visant à étendre un ensemble de garanties fondamentales de sécurité sociale par la création d'un socle de protection sociale, ainsi qu'une dimension verticale visant quant à elle à fournir un éventail plus large de prestations et à étendre la couverture sociale conformément aux conventions en vigueur relatives à la sécurité sociale, grâce à la mise en place d'une approche progressive ${ }^{38}$.

Ceci a entraîné l'adoption de la Recommandation n 202 de l'OIT sur les socles de protection sociale (2012), un instrument important au regard de l'extension de la protection sociale aux travailleuses vulnérables de l'économie informelle qu'il promeut. Cet instrument a été vu comme proposant une meilleure approche de l'extension de la sécurité sociale universelle, une approche plus réaliste pour les pays en développement. En ce sens, la Recommandation fournit des conseils aux États membres sur la création et le maintien de socles de protection sociale $^{39}$. Le préambule reconnaît la valeur de la sécurité sociale en tant qu'outil permettant de prévenir et de réduire la pauvreté, les inégalités, l'exclusion sociale et l'insécurité sociale, permettant de promouvoir l'égalité des chances, l'égalité entre hommes et femmes et l'égalité raciale et permettant finalement d'appuyer la transition entre le travail informel et le travail formel. Ce point est pertinent pour les travailleuses de l'économie informelle, qui sont souvent exclues de la couverture et se heurtent à des discriminations à plus d'un titre, telles que discriminations raciales et en fonction du sexe. Le préambule reconnaît également le lien évident qui existe entre des systèmes de sécurité sociale durables et le passage à l'économie formelle. L'article 3 recommande aux États membres d'appliquer des principes importants tels que la non-discrimination, l'égalité entre hommes et femmes et la prise en compte des besoins spécifiques. Il s'agit là d'un principe important pour les travailleurs de l'économie informelle, dont certains, tels que les travailleurs domestiques et à domicile, ont des besoins très spécifiques en matière de protection sociale.

Les socles de protection sociale doivent comprendre des garanties fondamentales de sécurité sociale, telles que les soins de santé essentiels ly compris les soins de maternitél, la sécurité élémentaire de revenu pour les enfants, assurant l'accès à l'alimentation, à l'éducation et aux soins, la sécurité élémentaire de revenu pour les personnes qui sont dans l'incapacité de gagner un revenu suffisant ${ }^{40}$, ainsi que la sécurité élémentaire de revenu pour les personnes âgées ${ }^{41}$. Lors de l'extension de la protection sociale à ces travailleurs, il convient de tenir compte des situations professionnelles et des différents besoins, et donc d'envisager diverses méthodes et approches pour financer et fournir ces prestations ${ }^{42}$.

38 OIT, Étude d'ensemble 2011 concernant les instruments relatifs à la sécurité sociale à la lumière de la Déclaration de 2008 sur la justice sociale pour une mondialisation équitable, Conférence internationale du Travail, 2011, $100^{\text {e }}$ session, p. 136.

39 Voir l'article 1.

40 Il s'agit des personnes d'âge actif qui sont dans l'incapacité de gagner un revenu suffisant, en particulier dans les cas de maladie, de chômage, de maternité et d'invalidité. Article 5.

41 Voir l'article 5.

42 Voir l'article 3. 
L'article 15 mentionne expressément l'intégration sociale des personnes issues de l'économie informelle. La Recommandation appelle à l'adoption de stratégies d'extension de la sécurité sociale afin de soutenir la croissance de l'emploi formel et la réduction de l'informalité. Les États membres sont responsables de la mise en œuvre, les organes de contrôle de l'OIT ne disposant que d'une marge de manœuvre limitée pour procéder au suivi des progrès ${ }^{43}$. L'OIT a fourni une assistance technique, appuyant ainsi la mise en place de socles de protection sociale dans plusieurs pays ${ }^{44}$.

Malgré son caractère non contraignant, la Recommandation a donné naissance à plusieurs initiatives importantes en matière d'extension de la protection sociale à des personnes précédemment exclues. Citons par exemple : au Ghana, un régime pilote d'allocations maternité destiné aux travailleuses de l'économie informelle a été mis en place ${ }^{45}$; l'Inde envisage de créer un ensemble de prestations sociales de base universelles; le Lesotho songe à instaurer un système national de santé (projet de loi relatif à l'assurance santé nationale); Madagascar a lancé un projet visant à étendre la couverture sociale, plus précisément les prestations chômage et maladie, aux personnes travaillant dans le secteur informel de l'agriculture et de la pêche, ainsi qu'à leur famille; la Namibie projette quant à elle d'adopter des garanties minimales en matière de sécurité de revenu et d'accès universel aux soins de santé ${ }^{46}$. D'autres pays membres de la SADC, comme la République-Unie de Tanzanie $^{47}$ et le Zimbabwe ${ }^{48}$, prévoient également d'adopter des mesures juridiques visant à étendre la protection. Ces exemples montrent que la santé représente un élément important pour ces pays qui s'efforcent d'étendre la protection à l'ensemble de leur population, et qu'elle constitue souvent le point de départ de l'extension de la protection sociale dans son ensemble.

43 U. Becker, F. Pennings et T. Dijkhoff (dir.), International Standard Setting and Innovations in Social Security, Kluwer Law International, 2013, p. 28.

44 L'OIT a aidé plus de 136 pays à mettre en place des socles de protection sociale, prenant la forme d'un régime de protection sociale nationale en Colombie, d'un régime de retraite sans cotisation au Pérou, de l'extension des prestations santé et retraite et des allocations familiales en Uruguay, et d'un régime d'allocations familiales universelles en Argentine. OIT, http://www.ilo.org/global/lang--fr/index.htm (consulté le 10 juin 2017).

45 Ce point se situe au sommet de la liste des priorités du Ghana. Le Rapport sur la santé dans le monde de 2005 ayant indiqué que $75 \%$ des décès néonatals pouvaient être évités si les femmes et les enfants bénéficiaient de soins adaptés pendant la grossesse et l'accouchement, ainsi que par la suite. Des organisations de membres et autres organisations internationales telles que la Fondation Rockefeller ont participé à l'extension des mesures de protection sociale aux populations les plus vulnérables du Ghana. OIT, http://www.ilo.org/global/lang--fr/index.htm (consulté le 10 juin 2017).

46 OIT, Étude d'ensemble 2011 concernant les instruments relatifs à la sécurité sociale à la lumière de la Déclaration de 2008 sur la justice sociale pour une mondialisation équitable, Conférence internationale du Travail, 2011, $100^{\mathrm{e}}$ session, p. 138-139.

47 L'introduction d'une pension universelle de vieillesse et d'un accès aux soins médicaux pour tous est à l'étude. OIT, Étude d'ensemble 2011 concernant les instruments relatifs à la sécurité sociale à la lumière de la Déclaration de 2008 sur la justice sociale pour une mondialisation équitable, Conférence internationale du Travail, 2011, $100^{\mathrm{e}}$ session, p. 140.

48 L'introduction d'un régime d'assurance-maladie universel est envisagée, OIT, Étude d'ensemble 2011 concernant les instruments relatifs à la sécurité sociale à la lumière de la Déclaration de 2008 sur la justice sociale pour une mondialisation équitable, Conférence internationale du Travail, 2011, $100^{\mathrm{e}}$ session, p. 140. 
Dans ses efforts visant à adopter un instrument flexible adapté aux pays en développement, l'OIT a omis de prévoir des critères généraux de suivi et d'évaluation des progrès effectués. Par ailleurs, il n'existe aucune obligation de présenter un rapport à l'OIT sur ce point ${ }^{49}$. La recherche d'un équilibre entre souplesse et normes minimales implique que les effets de la Recommandation sur l'extension de la protection sociale à tous demeurent discutables, mais illustre très certainement la situation délicate dans laquelle se retrouve l'OIT lorsqu'elle cherche à mettre en balance la diversité des intérêts des États membres et des travailleurs vulnérables à travers l'élaboration de normes internationales. Dans les pays dont la Constitution exige de prendre en compte et d'appliquer le droit international, comme l'Afrique du Sud, les instruments internationaux de sécurité sociale produisent davantage d'effets ${ }^{50}$.

Outre les instruments internationaux, il existe également des accords internationaux bilatéraux et multilatéraux de sécurité sociale. Ces accords visent souvent à étendre la couverture aux noncitoyens, et contiennent des principes fondamentaux tels que l'égalité de traitement, le maintien des droits acquis, le paiement des prestations à l'étranger, la détermination des droits acquis, le maintien des droits en cours d'acquisition, et la réciprocité ${ }^{11}$.

Les principaux instruments de sécurité sociale applicables aux travailleurs de l'économie informelle sont les suivants : la Convention $\mathrm{n}^{\circ} 102$ concernant la sécurité sociale (norme minimum) de 1952; la Convention $n^{\circ} 183$ sur la protection de la maternité de 2000, et la Convention $n^{\circ} 155$ sur la sécurité et la santé des travailleurs de 1981. Le taux de ratification de ces conventions par les pays de la SADC demeure faible.

La mise en œuvre des normes de l'OIT peut poser davantage de problèmes dans les pays en développement, qui ne sont pas nécessairement en mesure de respecter les obligations qui leur incombent. Toutefois, les gouvernements peuvent commencer par supprimer tous les obstacles à la liberté d'association de ces travailleurs ${ }^{52}$. Certaines conventions de l'OIT permettent aux pays les ayant ratifiées d'adopter des normes adaptées à la situation nationale. L'OIT ouvre la couverture aux travailleurs atypiques par le biais de conventions adaptées favorisant une réception plus universelle, la promotion et l'extension de la protection à ces travailleurs. Elle devrait envisager de lancer une campagne prioritaire ayant pour objet d'encourager les pays en développement, où prévaut l'économie informelle, à ratifier les conventions relatives à la protection sociale ${ }^{53}$. Grâce aux activités développées par ses bureaux dans les différents pays, l'OIT peut encourager le respect de ces instruments juridiques. Les normes internationales peuvent servir de feuille de route aux politiques et législations envisagées.

49 U. Becker, F. Pennings et T. Dijkhoff (dir.), International Standard Setting and Innovations in Social Security, Kluwer Law International, 2013, p. 28.

50 Voir les articles 39(1), 232 et 233 de la Constitution de la République d'Afrique du Sud, 1996.

51 G. Mpedi, «Introduction », in U. Becker, F. Pennings et T. Dijkhoff (dir.), International Standard Setting and Innovations in Social Security, op. cit., 2013, p. 5.

52 Le Bangladesh a dressé des obstacles inutiles à la liberté d'association, en exigeant qu'au moins $30 \%$ des travailleurs d'un établissement adhèrent à un syndicat avant que celui-ci ne puisse être valablement enregistré par le gouvernement - voir Human Rights Watch, httpw://www.hrw.org/news/2013/Bangladesh. Compte tenu des nombreux obstacles entravant la liberté d'association des travailleurs de l'économie informelle, les gouvernements doivent, pour s'acquitter correctement de leurs obligations internationales, supprimer les exigences les empêchant de s'organiser.

53 G. Mpedi, «Informal Sector : Introduction », in U. Becker, F. Pennings et T. Dijkhoff (dir.), International Standard Setting and Innovations in Social Security, Kluwer Law International, op.cit., 2013, Pays-Bas, p. 44. 


\section{III - Instruments régionaux : la Communauté de développement de l'Afrique australe (SADC)}

En 1992, les chefs d'État ont convenu de transformer l'ancienne SADCC en SADC, davantage axée sur l'intégration du développement économique ${ }^{54}$. La vision, fondée sur des principes et valeurs communes, est celle d'une communauté régionale au sein de laquelle les conditions de vie sont améliorées, la pauvreté réduite, et qui assure à tous la liberté, la justice sociale, la paix et la sécurité. L'article 3 du Traité de la SADC (1992) confère à la Communauté une personnalité juridique, ainsi que le statut d'organisation internationale ${ }^{55}$. Les signataires du Traité s'engagent à harmoniser leurs politiques, à prendre des initiatives pour resserrer les liens dans la région et à participer à la mise en œuvre de tous les projets et programmes de la Communauté. L'article 5 contient d'importants objectifs, tels que la réduction de la pauvreté, le renforcement du niveau et de la qualité de vie de la population, ainsi que l'appui aux personnes socialement défavorisées dans la région par le biais de l'intégration ${ }^{56}$. Les pays de la Communauté coopèrent en matière de sécurité sociale, de développement social et humain et de mise en œuvre de programmes spéciaux, essentiels pour assurer l'extension de la protection sociale aux travailleurs vulnérables ${ }^{57}$. L'article 4 du Traité de la SADC reconnaît le principe d'égalité souveraine au sein de la Communauté, ce qui signifie que les programmes adoptés ne seront pas applicables de façon automatique dans les États membres, lesquels ne pourront être forcés de mettre en œuvre l'ensemble de ces initiatives et projets sur leur territoire ${ }^{58}$. L'article 5 recense les mécanismes qui permettront aux États membres d'atteindre ces objectifs. Citons, par exemple, l'harmonisation des politiques, l'élaboration de politiques communes et la promotion du développement sur différents sujets pour aider les États membres à atteindre leurs objectifs. L'article 21(1) oblige les États membres à coopérer à tous les niveaux pour favoriser l'intégration régionale et le développement. Lorsque l'on étudie la situation difficile des travailleurs de l'économie informelle dans la région, il convient de s'attarder sur l'article 21(3) (f), qui affirme que le bien-être social, qui englobe sans aucun doute les notions de protection sociale, de travail décent pour tous et d'intégration sociale dans la région, constitue l'un des sujets requérant de la coopération ${ }^{59}$. Pour atteindre cet objectif, les États membres devront coopérer en vue de l'adoption d'un cadre juridique adapté, de la création d'un cadre institutionnel et de la mise en œuvre d'un cadre programmatique ${ }^{60}$.

54 Les États suivants sont membres de la SADC : Afrique du Sud, Angola, Botswana, Lesotho, Madagascar, Malawi, Maurice, Mozambique, Namibie, République démocratique du Congo, République-Unie de Tanzanie, Seychelles, Swaziland, Zambie et Zimbabwe. La SADC compte une population totale de 277 millions d'habitants.

55 Voir les articles 3(1) et (2).

56 Voir l'article 5(1).

57 L'Amendement au Traité de la SADC de 2009 a mis en place le cadre institutionnel suivant : Sommet des chefs d’État; Organe pour la politique, la défense et la coopération en matière de sécurité ; Conseil des ministres; Secrétariat; Tribunal; Troïka; Comité permanent des hauts fonctionnaires; Comité national de la SADC. Voir l'article 9.

58 G. Mpedi et M. Nyenti, «Perspectives towards the Development of the Social Protection Dimension of the SADC Regional Integration Agenda », SADC Law Journal, 2012, p. 166.

59 Ibidem.

60 Ibidem. 
Les pays de la SADC sont, dans une certaine mesure, interdépendants et reliés par des flux migratoires ${ }^{61}$. Il est important de noter que la majorité des travailleurs évoluent dans l'économie informelle, généralement en dehors du champ des protections en matière de sécurité sociale et de travail. La région présente également l'une des plus fortes prévalences du VIH et du sida au monde, qui peut s'expliquer par les facteurs suivants : pauvreté extrême, inégalités de revenu, mobilité des travailleurs migrants, dynamiques sexo-spécifiques et violence sexiste ${ }^{62}$. En juin 2016, le Secrétariat de la SADC a annoncé qu'environ 41 millions de personnes dans la région souffraient d'insécurité alimentaire ${ }^{63}$. Ce fait met en lumière la situation des groupes particulièrement vulnérables dans la région, comme les personnes vivant avec le VIH et le sida.

Les instruments régionaux au sein de la SADC peuvent jouer un rôle important dans l'extension des protections en matière de sécurité sociale et de travail au profit des travailleurs de l'économie informelle. Les textes pertinents sont : le Traité de la SADC ${ }^{64}$, la Charte des droits sociaux fondamentaux ${ }^{65}$, le Code de la sécurité sociale ${ }^{66}$, l'avant-projet de Protocole sur la facilitation de la circulation des personnes ${ }^{67}$, la Déclaration sur le Genre et le développement ${ }^{68}$, le Protocole relatif à la santé ${ }^{69}$, le Code de conduite à l'égard du VIH et du sida et de l'emploi dans la SADC70 et le Protocole sur l'éducation et la formation ${ }^{71}$.

\section{IV - Expression et représentation collectives}

La Résolution de l'OIT concernant le travail décent et l'économie informelle de 2002 reconnaît que les travailleurs de l'économie informelle, salariés comme indépendants, ont les mêmes droits à un travail décent et à une représentation que les travailleurs de l'économie formelle, et que les gouvernements doivent établir un cadre national et local favorisant la concrétisation de leur droit à la représentation. La conclusion $n^{\circ} 9$ insiste sur le fait que, n'étant généralement pas organisés, ces travailleurs ne sont pas, ou quasiment pas, représentés collectivement «auprès des employeurs ou des autorités publiques».

61 M. Olivier et E. Kalula, «Regional Social Security», in M. Olivier, N. Smit et E. Kalula, Social Security: A Legal Analysis, 2003, Lexis Nexis South Africa, p. 660-661.

62 SADC, http://www.sadc.int (consulté le 10 mars 2017).

63 SADC, http://www.sadc.int (consulté le 10 mars 2017).

64 (1992).

65 (2003). L'un des principaux objectifs de la Charte est d'aligner les politiques et programmes juridiques, économiques et sociaux, de créer des régimes de sécurité sociale et de les harmoniser. La Charte reconnaît les droits fondamentaux, l'égalité pour tous et le besoin de protection des groupes vulnérables. Son article 10 impose aux États membres de créer un environnement propice fournissant à chaque travailleur une protection sociale adaptée, quel que soit son statut.

66 (2007). Ce code concerne les migrants employés légalement et appelle à leur protection. Il couvre également les migrants ayant le statut de travailleur indépendant. Il fournit, par ailleurs, des directives sur le développement, la coordination et la consolidation de la sécurité sociale.

67 (2005)

68 (1997).

69 (1999).

70 (1997).

71 (1997). 
Il est particulièrement difficile pour les syndicats d'atteindre les travailleurs de l'économie informelle; ce problème est urgent et ne peut être ignoré. Les syndicats doivent s'engager dans des négociations sociales et politiques avec les autorités publiques et les organes législatifs. Les dispositions législatives actuelles prévoient généralement des structures et procédures de négociation collective fondées sur les relations de travail traditionnelles. Toutefois, les travailleurs de l'économie informelle ont souvent affaire à différentes entités de négociation, comme les municipalités, les représentants du gouvernement et d'autres organes décisionnels ayant une emprise directe sur leurs moyens de subsistance. Les syndicats se heurtent à des difficultés politiques et conceptuelles, à l'absence de ressources et d'expérience, voire de volonté politique d'organiser les travailleurs de l'économie informelle.

La Self-Employed Women's Association (SEWA) en Inde a une importante leçon à donner : l'absence de rapport apparent entre employeur et travailleur, qui caractérise souvent l'économie informelle, signifie que les stratégies habituellement adoptées sur le lieu de travail ne permettront pas d'organiser les travailleurs de l'économie informelle ${ }^{72}$. Les difficultés appellent des solutions innovantes, telles que de nouvelles stratégies d'organisation, des moyens d'action adaptés, de nouvelles structures, des outils de communication innovants ou encore des formes d'actions collectives efficaces. Les travailleurs de l'économie informelle peuvent être approchés par le biais d'ONG et d'autres organes de la société civile. Pour être durables, les organisations œuvrant en faveur de ces travailleurs doivent bénéficier de l'appui de structures politiques et de stratégies reconnaissant la contribution majeure qu'apportent les travailleurs pauvres ${ }^{73}$.

En Afrique du Sud, les travailleurs domestiques jouissent de la liberté d'association ${ }^{74}$. Le syndicat South African Domestic Service and Allied Workers Union (SADSAWU) ${ }^{75}$, le plus grand syndicat de travailleurs domestiques du pays à l'heure actuelle ${ }^{76}$, représente cette population dans des villes comme Le Cap, Johannesburg et Durban. Créé en 2000, il fait suite à la South African Domestic Workers Association (SADWU), elle-même fondée en 1986. Sa présidente, Myrtle Witbooi, a travaillé comme domestique sous le régime de l'apartheid. Il convient d'étudier la mobilisation et le militantisme en faveur des droits du travail à la lumière de certains événements historiques. Le Congress of South African Trade Unions a vu le jour en 1955. Cinq ans plus tard, en 1960, le gouvernement de l'époque interdisait les organisations politiques. Malgré les manifestations de femmes contre les lois de 1956 sur les laissez-passer, en particulier la loi applicable aux travailleurs domestiques, toutes les femmes noires ont été contraintes de posséder un laissez-passer en 1964.

72 L. Vryenhoek, « Headstrong: Profile of a Headloader in Ahmedabad, India », WIEGO Workers' Lives n² 2012, p. 3

73 C. Mather, Informal Workers' Organizing, Rapport de recherche WIEGO, 2012, p. 12.

74 Article 4. L'article 23 al. 2, 3 et 4 de la Constitution accorde aux travailleurs les droits fondamentaux de former et de s'affilier à des syndicats, de faire grève et de prendre part à leurs activités. L'article 23 al. 5 reconnaît le droit d'entamer des négociations collectives. Ces droits sont sous-tendus par la liberté de réunion et les droits de manifester, de faire grève et de formuler des revendications de façon pacifique, reconnus par l'article 17, et par la liberté d'association contenue dans l'article 18.

75 SADSAWU, http://www.sadsawu.com (consulté le 10 juin 2017).

76 En Tanzanie, les travailleurs domestiques sont affiliés à un syndicat plus important, « Conservation », qui s'attache à renforcer leurs capacités pour favoriser leur autonomisation. N. Vanqa, Y. Wiid et D. Du Toit, «Organizing for Empowerment», in D. du Toit (dir.), Exploited, Undervalued - and Essential: Domestic Workers and the Realisation of their Rights, Pretoria University Law Press, 2013, p. 283. 
Les grèves syndicales menées à partir de 1973 ont servi d'outil puissant de résistance politique contre le régime politique. Dès 1980, les travailleurs domestiques ont commencé à faire campagne pour l'éducation et l'obtention de conditions de travail décentes. Le SADSAWU fournit également une assistance aux employeurs pour les aider à respecter les dispositions législatives.

Les différents contextes sociaux, culturels, religieux et politiques peuvent avoir un effet sur la mobilisation des travailleuses de l'économie informelle. En Inde, elles ont pu vouloir s'opposer au recours à l'action collective, en raison de l'épuisement, de la victimisation et du poids des responsabilités familiales qu'elles supportent ${ }^{77}$. Elles sont souvent conditionnées par leurs communautés à ne pas s'affirmer à cause de stéréotypes sexistes et d'attitudes paternalistes inégalitaires, victimisées et soumises à des violences sexistes ${ }^{78}$.

Les syndicats et organisations de travailleurs peuvent largement contribuer à l'extension de la protection aux travailleuses informelles, en les faisant bénéficier des dispositions législatives en vigueur et/ou en concevant des solutions innovantes et adaptées. Il est en outre évident que, pour parvenir à organiser les travailleuses de l'économie informelle, les syndicats devront militer en faveur d'une approche globale, et leur collaboration avec d'autres parties prenantes devra donc porter également sur d'autres droits fondamentaux pour assurer une protection adaptée. Ceci signifie, toutefois, que le rôle des syndicats doit évoluer, non seulement pour couvrir les négociations salariales avec l'employeur, mais aussi pour entamer des négociations sociales et politiques avec les autorités publiques et les organes législatifs. Les travailleurs de l'économie informelle ont souvent affaire à différentes entités de négociation, comme les municipalités, les représentants du gouvernement et d'autres organes décisionnels, ayant une emprise directe sur leurs moyens de subsistance. Ceci suppose d'identifier les partenaires de négociation et d'établir une relation dotée d'une vision à long terme.

L'adoption de pratiques innovantes, la mise en place de nouvelles stratégies d'organisation et la coopération avec des organisations communautaires et de travailleurs permettront aux travailleuses de l'économie informelle d'être mieux représentées, d'améliorer leurs conditions de travail et, au bout du compte, de transformer leur existence. Les dispositions législatives pourraient être étendues, de façon à mettre en place des structures visant à satisfaire les besoins de ces personnes, et notamment un processus de résolution des litiges conçu à cette fin.

Assurer le respect des droits fondamentaux ne suffit pas : les gouvernements devraient se rapprocher des syndicats et des organisations de travailleurs qui représentent cette population, et mettre en place des mesures innovantes visant à répondre à leurs besoins particuliers. Ils devraient, par exemple, prévoir des procédures d'enregistrement simplifiées, plus rapides et moins chères pour ces organisations. Les syndicats existants peuvent faire tout ce qui est en leur pouvoir pour accueillir et protéger les travailleurs d'une économie informelle en plein essor, mais les syndicats indépendants créés par ces derniers seront plus à même de se concentrer sur les questions les plus pertinentes pour eux. Une bonne gouvernance dénuée d'actes de harcèlement, de corruption et d'extorsion de la part de fonctionnaires peu scrupuleux est essentielle pour faciliter l'accès des travailleurs de l'économie informelle aux ressources, informations, marchés, infrastructures publiques, technologies et autres mesures de protection sociale.

77 J. Jenkins, « Organizing “Spaces of Hope”: Union Formation by Indian Garment Workers », BJIR, 2013, p. 633.

78 J. Jenkins, «Organizing “Spaces of Hope”: Union Formation by Indian Garment Workers », BJIR, 2013, p. 633. 


\section{V - Exemples de mesures législatives visant à étendre la protection sociale aux travailleurs de l'économie informelle}

\section{A - Afrique du Sud}

Les travailleurs domestiques sont désormais couverts par la quasi-totalité des lois relatives au travail en Afrique du Sud, grâce à un règlement ministériel sectoriel relatif à l'article 51 de la loi 75 sur les conditions fondamentales d'emploi (BCEA ci-après), de 1997. Ce règlement s'applique à tous les travailleurs et travailleuses domestiques d'Afrique du Sud, y compris les entrepreneurs indépendants et salariés mis à disposition par les services de l'emploi ${ }^{79}$. Ce règlement régit les salaires, les heures de travail, les congés et les conditions de cessation du contrat de travail, et étend les dispositions de la BCEA aux travailleurs domestiques.

Les travailleurs domestiques employés sur les exploitations agricoles et effectuant des travaux agricoles, ainsi que les travailleurs domestiques couverts par une autre réglementation, tels que les ouvriers agricoles ou les travailleurs protégés par un accord conclu au sein d'un conseil de négociation, sont exclus du champ d’application du règlement. Les clauses 2 et 3 du règlement régissent les questions du salaire et de ses augmentations. Il s'agit des deux seules clauses applicables aux travailleurs domestiques qui travaillent moins de 24 heures par mois pour un même employeur, ce qui pose problème et entre en contradiction avec les progrès constitutionnels en matière de justice sociale ou les obligations internationales relatives au travail décent pour tous contractées par le pays. Souvent, les travailleurs domestiques ont plusieurs employeurs, mais travaillent moins de 24 heures pour chacun d'entre eux. Ils sont donc exclus des protections du travail, et leurs employeurs n'ont aucune obligation de cotiser à l'assurance chômage.

Ces dispositions favorisent potentiellement l'exploitation des travailleurs vulnérables, les employeurs pouvant délibérément choisir d'employer des travailleurs pour une durée inférieure à 24 heures afin d'éviter les clauses susmentionnées. Cette exclusion va donc à l'encontre du but visé par la loi, à savoir protéger les travailleurs vulnérables ${ }^{80}$.

Les lois relatives à l'assurance chômage ${ }^{81}$ (UIA) et aux cotisations à l'assurance chômage ${ }^{82}$ (UICA) couvrent les travailleurs domestiques depuis 2003. Les employeurs et les salariés sont tenus de cotiser à hauteur de $1 \%$ de la rémunération mensuelle perçue par le travailleur ${ }^{83}$. En cotisant, les salariés qui se retrouvent au chômage ont le droit de percevoir un ensemble de

79 Clause 1. Les travailleurs domestiques des Philippines sont également protégés par un règlement sectoriel qui fixe un salaire minimum dépendant du lieu de travail. Au Brésil, la règlementation relative au salaire minimal national s'applique aux travailleurs domestiques. En Inde, ce sont les différents États qui ont compétence pour appliquer ou non le seuil de salaire minimum aux travailleurs domestiques, la loi sur le salaire minimum ne faisant pas expressément référence au travail domestique. OIT, «Domestic Work, Wages, and Gender Equality: Lessons from Developing Countries», document de travail n 7, 2015, p. 11.

80 D. Du Toit et E. Huysamen, «Implementing Domestic Workers' Labour Rights in a Framework of Transformative Constitutionalism», in D. du Toit (dir.), Exploited, Undervalued-and Essential: Domestic Workers and the Realisation of their Rights, Pretoria University Law Press, 2013, p. 83.

81 Loi 63 de 2001

82 Loi 4 de 2002. Cette loi définit le «travailleur domestique» comme un travailleur effectuant un travail domestique au domicile de son employeur, à savoir : (a) les jardiniers; (b) les personnes employées comme conducteur d'un véhicule motorisé; et (c) les personnes prenant soin d'une autre personne au domicile de leur employeur. La même définition figure dans la Loi 63 sur l'assurance chômage de 2001, article $1 \mathrm{er}$.

83 Article 6 de l'UICA. 
prestations listées dans l'article 12 de l'UIA, à savoir des allocations chômage ${ }^{84}$, des indemnités maladie $^{85}$, des allocations de maternité ${ }^{86}$, des allocations d'adoption $^{87}$ et des indemnités pour personnes à charge ${ }^{88}$. La loi contient des dispositions spéciales applicables aux travailleurs domestiques ayant plusieurs employeurs : le licenciement par un employeur leur ouvre des droits à des prestations même s'ils conservent leur emploi chez les autres employeurs ${ }^{89}$.

\section{B - Zambie ${ }^{90}$}

Le programme social d'allocations en espèce compte 242000 bénéficiaires. Le système de sécurité sociale actuel est fragmenté, ce qui a poussé le gouvernement à mettre en place un système national de sécurité sociale complet. En 2014, le gouvernement s'est engagé, dans un document stratégique, à mettre en place un régime de protection sociale exhaustif, dont le cadre juridique sera le projet de loi sur la protection sociale. Ce dernier prévoira l'extension progressive des mesures de protection sociale aux travailleurs actuellement exclus. Il envisage la mise en place d'un système souple répondant aux diverses caractéristiques de ces travailleurs. Plusieurs difficultés liées à l'extension des mesures de protection sociale aux travailleurs de l'économie informelle ont été identifiées : des revenus irréguliers et faibles; une absence de confiance dans le cadre institutionnel; un manque de connaissances sur les lois, institutions et procédures existantes; l'absence ou la faiblesse des organisations de travailleurs; l'isolement et la division; et l'exclusion actuelle des systèmes.

Le gouvernement fait preuve de volonté politique en établissant une autorité unique chargée de la protection sociale, ayant pour mission de fournir des prestations sociales complètes à l'ensemble de la population. En ce qui concerne les travailleurs de l'économie informelle, une stratégie nationale, qui peut être adaptée aux besoins particuliers de différentes catégories de travailleurs informels, tels que les travailleurs indépendants ou les travailleurs domestiques, est envisagée.

Un ensemble unique de prestations sociales fondé sur des cotisations unifiées est proposé. Reconnaissant les difficultés auxquelles font face ces travailleurs et le fait que les besoins immédiats sont souvent priorisés, il est possible d'effectuer des dépenses sans attendre.

84 Article 12(1)(a). Un cotisant a droit à des indemnités chômage lorsque le chômage est dû à l'expiration d'un contrat à durée déterminée, à un licenciement ou à l'insolvabilité de l'employeur. Article 16(a).

85 Article $12(1)(b)$. L'article 20 prévoit que le travailleur cotisant a droit à ces prestations lorsqu'il est dans l'incapacité de travailler en raison d'une maladie, si celle-ci s'étale sur une durée supérieure à sept jours. Voir l'article 20.

86 Article $12(1)(c)$. La durée du congé maternité est fixée à 17,32 semaines. Des dispositions spéciales s'appliquent en cas de fausse-couche ou de naissance d'un enfant mort-né au cours du troisième trimestre.

87 Article 12(1)(d). Les adoptions doivent être conformes aux dispositions de la loi 74 sur la garde d'enfants de 1983.

88 Article 12(1)(e). Ces dispositions visent le conjoint ou le partenaire survivant, et les enfants dépendants en l'absence de conjoint ou de partenaire survivant. Article 30.

89 Article 12(1A).

90 M.P. Goursat et L. Pelterano, Extension of Social Protection to Workers in the Informal Economy in Zambia Lessons Learnt from Field Research on Domestic Workers, Small Scale Farmers and Construction Workers, OIT, 2016, p. 1-41. 
Dans le cadre du nouveau système proposé, malgré la grande mobilité professionnelle des travailleurs informels, ceux-ci bénéficieront de la portabilité des prestations, leur garantissant ainsi une couverture sociale. Ce nouveau système vise à dépasser les difficultés relatives aux cotisations, en imposant une contribution forfaitaire aux indépendants, modulée en fonction du nombre de membres de leur foyer. En ce qui concerne les travailleurs de l'économie informelle ayant des revenus irréguliers, les stratégies proposées introduiront le prélèvement de taxes sur un emploi ou un projet particulier. Ce système favorisera les cotisations mensuelles ou périodiques adaptées aux capacités des différentes catégories de travailleurs. L'introduction de ce système de protection sociale complet s'accompagnera de programmes d'autonomisation et de campagnes de promotion.

Des mesures incitatives spécifiques destinées aux ménages employant des travailleurs domestiques, aux travailleurs indépendants et aux petites entreprises seront adoptées. Le gouvernement compte mettre en œuvre un programme national de protection de la maternité à partir de 2018. Il est également important d'observer que, conformément au document politique, il s'est engagé à analyser la situation de l'économie informelle afin de déterminer les bonnes pratiques à mettre en œuvre pour favoriser l'extension des mesures de sécurité sociale. Le programme de protection sociale exhaustif envisagé par l'État est fondé sur les valeurs d'égalité et de dignité humaine ${ }^{91}$.

\section{Conclusion}

Il n'existe pas de solution immédiate au problème de la fourniture d'une protection en matière de sécurité sociale et de travail adaptée aux travailleuses vulnérables. L'économie informelle se développe et le taux important de participation à ce secteur constitue la norme dans les pays en développement. Les travailleurs de l'économie informelle sont vulnérables et ne bénéficient pas de la protection juridique qu'ils méritent, puisqu'ils sont souvent exclus du champ d'application des dispositions législatives en vigueur. Si l'OIT reconnaît les nouvelles formes de travail dans ses normes, le taux de ratification des conventions applicables demeure faible et la mise en œuvre des normes de l'OIT favorables aux travailleurs dans les pays en développement demeure problématique.

91 Politique nationale de protection sociale, ministre du Développement communautaire et de la Santé maternelle et de l’enfant, République de Zambie, 2014, p. 28. 


\section{L'ÉCONOMIE INFORMELLe en Afrique Australe}

Les travailleurs de l'économie informelle doivent s'organiser pour améliorer et transformer leurs conditions de vie. Toutefois, les syndicats ont toutes les difficultés du monde à représenter et organiser l'économie informelle. Les gouvernements devraient créer un cadre favorable soutenant les politiques et programmes de l'économie informelle et d'autres institutions, telles que les associations, les coopératives, les ONG et les organisations communautaires, qui peuvent établir des liens à l'échelle nationale et internationale et aider à promouvoir les intérêts des travailleurs.

Il est parfois avancé que l'économie informelle n'est pas amenée à disparaître de sitôt, et qu'elle ne sera pas absorbée par l'économie formelle, mais que la fourniture d'un emploi aux pauvres et la réduction de la pauvreté des ménages prendront toujours le dessus dans les pays en développement. Les normes internationales et régionales doivent être promues, et les gouvernements doivent fournir un soutien, des cadres favorables et des infrastructures aux travailleurs de l'économie informelle.

\section{ELMARIE FOURIE}

Maître de conférences à la Faculté de Droit de l'Université de Johannesburg.

Thèmes de recherche : Droit du travail, sécurité sociale, formation juridique.

\section{Parmi ses publications :}

"Exploring innovative solutions to extend social protection to vulnerable women workers in the informal economy", ILJ, 2016, pp. 831-846.

"Constitutional values, therapeutic jurisprudence and legal education in South Africa: Shaping our leagl order", PER, 2016, pp. 1-26. 